\title{
Effects of Pedunculopontine Tegmental Nucleus Lesions on Responding for Intravenous Heroin under Different Schedules of Reinforcement
}

\author{
Mary C. OImstead, ${ }^{1}$ Elizabeth M. Munn, ${ }^{2}$ Keith B. J. Franklin, ${ }^{3}$ and Roy A. Wise ${ }^{2}$ \\ ${ }^{1}$ Department of Psychology, Queen's University, Kingston, Ontario K7L 3N6, Canada, ${ }^{2}$ Centre for Studies in Behavioral \\ Neurobiology, Concordia University, Montréal, Québec H3G 1M8, Canada, and ${ }^{3}$ Department of Psychology, McGill \\ University, Montréal, Québec H3A 1B1, Canada
}

The pedunculopontine tegmental nucleus (PPTg) is believed to play important roles in reward and learning. We examined the effect of PPTg lesions $(0.5 \mu \mathrm{l}$ of $0.1 \mathrm{~m}$ NMDA injected bilaterally over $10 \mathrm{~min}$ ) on the learning of an operant response for opiate reward. In 14 adult male Long-Evans rats, bilateral lesions of the PPTg disrupted the acquisition of responding for intravenous heroin $(0.1 \mathrm{mg} / \mathrm{kg}$ infused at a rate of $0.25 \mathrm{ml} / 28 \mathrm{sec})$ on a fixed ratio-1 (FR-1) schedule of reinforcement. The 12 remaining lesioned animals increased their heroin intake over the acquisition sessions but did not reach the response levels of sham-lesioned animals on the 15th and final session. The sham- and PPTg-lesioned animals that learned the FR-1 task exhibited similar patterns of responding during extinction and reacquisition sessions. When tested on a progressive ratio (PR) schedule of reinforcement, however, PPTg-lesioned animals had lower break points than sham-lesioned animals. Asymmetric lesions, which destroyed the majority of the nucleus in one hemisphere only, did not produce any behavioral deficits. Rats that were lesioned after training also did not show deficits in responding under either FR or PR schedules. These findings suggest that PPTg lesions reduce the rewarding effect of opiates but do not disrupt the ability either to learn an operant response or the response requirements of a PR schedule.

Key words: brainstem; learning; opiates; PPTg; reward; self-administration
The pedunculopontine tegmental nucleus (PPTg) has been associated with a variety of diverse behavioral functions (for review, see Inglis and Winn, 1995). Based on a series of studies examining the neural interface between limbic and motor systems and how it relates to the generation of motivated behaviors, it was suggested that the PPTg is one element in a neural circuit that mediates limbic influences on the locomotor component of biologically significant behaviors (Mogenson et al., 1980; Mogenson, 1987, 1990). Previously, the ventral tegmental area (VTA) and its projections to forebrain limbic structures, particularly the ventral striatum (VS), were implicated in motivation in that electrical stimulation of these regions elicits a number of goal-directed behaviors (Glickman and Schiff, 1967). The VS projects via the ventral pallidum (VP) to the PPTg (Swanson and Cowan, 1975; Swanson et al., 1984), which in turn projects to the spinal cord through the nucleus reticularis gigantocellularis (Orlovsky, 1970; Garcia-Rill et al., 1983; Steeves and Jordan, 1984). Signals from other limbic sites may also be relayed through the VP to the PPTg, because more than two-thirds of the VP neurons antidromically activated by stimulation of the PPTg are inhibited by hippocampal stimulation and activated by amygdala stimulation (Tsai et al., 1989). Recent evidence indicates that the PPTg is even more intimately connected with forebrain limbic sites than

Received April 29, 1997; revised April 9, 1998; accepted April 9, 1998.

This work was supported by grants from the National Institute of Drug Abuse, the Natural Sciences and Engineering Research Council of Canada, and Fonds pour la Formation de Chercheurs et L'Aide à la Recherche du Québec. We thank Prof. Barry Everitt for helpful comments on earlier versions of this manuscript.

Correspondence should be addressed to M.C. Olmstead, Department of Psychology, Queen's University, Kingston, Ontario K7L 3N6, Canada.

Copyright (C) 1998 Society for Neuroscience $\quad 0270-6474 / 98 / 185035-10 \$ 05.00 / 0$ previously thought: efferents from the caudal region of the PPTg terminate in the VTA (Sugimoto and Hattori, 1984; Oakman et al., 1995b), and activation of PPTg neurons increases dopamine (DA) release in the VS (Klitenick and Kalivas, 1994). Finally, the finding that locomotion induced by injections of DA into the nucleus accumbens or picrotoxin into the VP is significantly reduced when synaptic transmission in the $\mathrm{PPTg}$ is blocked (Mogenson and Yang, 1991) supports Mogenson's proposal that the PPTg may be the site wherein emotional signals associated with motivated behaviors are translated into action (Mogenson et al., 1980).

Subsequent behavioral studies provided further evidence that the PPTg is involved in motivation and suggested that the nucleus may be part of a neural circuit that mediates reward. PPTg lesions block the development of conditioned place preferences (CPP) to opiates, stimulants, or food (Bechara and van der Kooy, 1989, 1992; Olmstead and Franklin, 1993, 1994a) and disrupt responding for intracranial stimulation (ICS) of the lateral hypothalamus (LH) (Buscher et al., 1989; Lepore and Franklin, 1996). PPTg lesions also disrupt feeding elicited by LH stimulation (Trojniar and Staszewska, 1995) and increase the frequency required to maintain ICS of the LH or VTA (Waraczynski and Shizgal, 1995). Likewise, disruption of cholinergic transmission from the PPTg to the VTA reduces the rewarding value of lateral hypothalamic self-stimulation (Yeomans et al., 1985, 1993; Kofman and Yeomans, 1989; Kofman et al., 1990).

In addition to its association with neural sites that mediate reward, the PPTg has connections with structures known to be involved in cognitive functions such as attention, learning, and memory. Cholinergic efferents from the PPTg innervate all of the thalamic nuclei (Hallanger et al., 1987; Rye et al., 1987; Newman 
and Ginsberg, 1994), project to the cholinergic neurons of the basal forebrain (Woolf and Butcher, 1986; Hallanger and Wainer, 1988), and control basal firing of acetylcholine (ACh) neurons of the nucleus basalis of Mynert (Bertorelli et al., 1991). It is not surprising, therefore, that PPTg lesions produce deficits in a variety of learning and memory tasks. For example, PPTg lesions impair the acquisition of active and passive avoidance (Fujimoto et al., 1989, 1992), disrupt performance in the radial arm and water maze tasks (Dellu et al., 1991; Lepore, 1993), and produce delay-dependent deficits in a spatial memory task (Kessler et al., 1986). Finally, although PPTg-lesioned animals can acquire responding for a conditioned reinforcer, they appear unable to discriminate between the levers, which do and do not produce the conditioned reinforcer (Inglis et al., 1994).

We assessed the role of the PPTg in reward and learning by examining the effect of PPTg lesions on the acquisition and maintenance of responding for intravenous heroin. The role of the PPTg in the rewarding effect of opiates in the CPP paradigm has been studied extensively by Bechara and colleagues (Bechara and van der Kooy, 1989, 1992; Bechara et al., 1992; Nader et al., 1994). They have demonstrated that PPTg lesions block the development of a CPP to morphine or heroin in drug-naive animals and that lesions are ineffective when they are made after conditioning sessions or when animals are drug-experienced. These results suggest that repeated drug administration and/or learning may compensate for the disruption of opiate-induced reward after PPTg lesions. To further examine this possibility, we considered three issues: the effects of pre- versus post-training lesions, the effects of lesions on responding under fixed and progressive ratio (PR) schedules of reinforcement, and the effects of lesions on responding during extinction and reacquisition.

\section{MATERIALS AND METHODS}

\section{Subjects and surgery}

Sixty-six adult male Long-Evans rats (Charles River, St. Constance, Québec, Canada) were anesthetized with sodium pentobarbital $(65 \mathrm{mg}$ / $\mathrm{kg}$, i.p., with $0.12 \mathrm{mg} / \mathrm{kg}$ atropine sulfate, s.c.) and secured in a stereotaxic apparatus. Animals received PPTg lesions before (pre-training) or after (post-training) self-administration training. Pre-training lesions $(n=55)$ were induced by bilateral injections of $0.1 \mathrm{M}$ NMDA dissolved in physiological saline $(0.5 \mu \mathrm{l}$ of a $7.2-7.6 \mathrm{pH}$ solution injected through 30 ga stainless steel tubing over $10 \mathrm{~min}$ ). Sham-lesioned animals received physiological saline infusions. The injector was left in place for $10 \mathrm{~min}$ after the infusion. Coordinates for the PPTg were $7.8 \mathrm{~mm}$ posterior to bregma, $1.6 \mathrm{~mm}$ lateral to the midline, and $7.2 \mathrm{~mm}$ ventral to the skull surface according to the atlas of Paxinos and Watson (1986). Animals that received post-training lesions $(n=11)$ were implanted with bilateral guide cannulae (23 ga) $1 \mathrm{~mm}$ dorsal to the injection site. After training, animals were anesthetized and received inf usions of the NMDA solution or vehicle (volume and concentration as above) through an injector that extended $1 \mathrm{~mm}$ beyond the guide cannulae.

After the lesion procedure or cannulae implantation, both sham- and PPTg-lesioned animals were implanted with chronically indwelling intravenous catheters. A SILASTIC catheter (Dow Medical Grade Tubing, Montréal, Québec, Canada; outer diameter, $1.2 \mathrm{~mm}$ ) was inserted into the right external jugular vein and secured with thread so that the tip reached the right atrium. The other end of the catheter was passed subcutaneously to an incision on the top of the skull. The catheter was connected to a bent 22 ga stainless steel cannula (Plastic Products, St. Albans, VT; model C313G) and attached to the skull with dental acrylic anchored by stainless steel screws. The cannula was used to connect the intravenous infusion line during self-administration sessions. The catheter was flushed with heparinized saline (200 USP units in $0.2 \mathrm{ml}$ of saline) and capped daily. Sterile penicillin G-procaine (Wyerth-Ayerst Canada, Inc.) was administered prophylactically after surgery $(60,000$ USP units in a volume of $0.2 \mathrm{ml}$ ). Both sham- and PPTg-lesioned animals were allowed to recover for 1-2 weeks before behavioral testing began.

\section{Procedure}

All animals were trained to self-administer heroin in operant cages $(26 \times$ $26 \times 28 \mathrm{~cm}$ ) enclosed in individually ventilated chambers. Before the self-administration sessions, the animals were drug-naive and had no experience with operant training. During self-administration sessions, each lever press led to an infusion of heroin $(0.1 \mathrm{mg} / \mathrm{kg}$ mixed in physiological saline) in a volume of $0.25 \mathrm{ml}$ over $28 \mathrm{sec}$. During the $28 \mathrm{sec}$ infusion, a light bulb located above the operative lever was lit, and bar presses were recorded but did not lead to further inf usions. The animals were tested during the active period of their circadian cycle. Water, but not food, was available during testing.

Pre-training lesions. Fifty-five animals received PPTg infusions before self-administration training. These animals were allowed to lever press for intravenous heroin on a fixed ratio-1 (FR-1) schedule of reinforcement for $4 \mathrm{hr} / \mathrm{d}$ for $15 \mathrm{~d}$. Three groups of animals were trained in this manner, and those that had viable catheters at the completion of the 15 acquisition sessions were subsequently tested under different conditions. The first group $(n=23)$ was tested for 5 d during extinction (syringes filled with saline) and then for $5 \mathrm{~d}$ under reacquisition conditions (syringes again filled with heroin). The second group $(n=20)$ was tested on a PR schedule of reinforcement for $5 \mathrm{hr} / \mathrm{d}$. Under the PR schedule, response requirements for successive heroin infusions were increased according to a formula established by Roberts and Bennett (1993). The animals continue to increase their number of responses for each injection of heroin until their behavior extinguishes. The last ratio repeated before responding ceased was defined as the break point. On each day, animals started the schedule two steps below the final ratio that was reached on the previous session. Testing continued until each animal's break point had stabilized $(<10 \%$ variation in the final ratio for four consecutive sessions). Using this procedure, animals must make an increasing number of lever presses to receive the first infusion of drug on successive sessions. When break points have stabilized, they are receiving an average of three infusions per session. The third group $(n=4)$ of rats that failed to acquire self-administration on the FR-1 schedule were injected daily with heroin $(0.5 \mathrm{mg} / \mathrm{kg}$, s.c. $)$ for $4 \mathrm{~d}$, and their operant responses on the same schedule were examined for an additional $15 \mathrm{~d}$. Heroin was injected at the end of each of these sessions.

Post-training lesions. Eleven animals with surgically implanted guide cannulae were trained to respond for heroin on an FR-1 schedule and were then switched to a PR schedule of reinforcement. After their responding had stabilized, they received bilateral lesions of the PPTg. After recovery from the lesions (1-2 weeks), these animals were retested on a PR schedule of reinforcement until their break point was stable ( $<10 \%$ variation in the final ratio for four consecutive sessions).

\section{Histology}

After behavioral testing was completed, all rats were anesthetized and perfused transcardially with physiological saline. The brains were immediately removed and frozen. Coronal sections (50 um) were cut and mounted on gelatin-coated slides. Alternate sections were stained with nicotinamide adenine dinucleotide phosphate (NADPH)-diaphorase, which marks neurons able to synthesize nitric oxide (Vincent et al., 1983). The strong correlation between the presence of nitric oxide and choline acetyltransferase in the mesopontine indicates that this histochemical stain may be used as a marker for cholinergic neurons in the PPTg (Vincent et al., 1983). Cholinergic neurons were counted using a Leitz DMRB microscope (Leica, Milton Keynes, UK) and an automated, quantitative image analysis system with software (Color Vision; Improvision, Coventry, UK) running on a Power Macintosh 8200 computer.

The remaining sections from PPTg-lesioned brains and a subpopulation of sham-lesioned brains $(n=10)$ were stained with cresyl violet to verify the placement and extent of the lesion-induced damage. The parameters used to define the PPTg anatomically have been the subject of some debate (for review, see Inglis and Winn, 1995). In the present study, the PPTg definition is based on the stereotaxic atlas of Paxinos and Watson (1986). Neuronal damage within subregions of the PPTg was determined using a grid analysis technique (Herberg and Franklin, 1972; Keesey and Powley, 1973) that assesses cell damage through successive anterior-posterior (A-P) planes of the nucleus. The majority of the PPTg cell volume falls within 6.72 to $8.72 \mathrm{~mm}$ posterior to bregma. The lateral and dorsoventral coordinates of the nucleus vary along its A-P axis (i.e., it spreads laterally and ventrally toward the posterior end), but both the width and depth are $\sim 1 \mathrm{~mm}$ within this $2 \mathrm{~mm} \mathrm{A-P}$ range. For each animal, the lesion-induced damage was represented on reproductions of plates 47-51 (Paxinos and Watson, 1986). Neuronal damage 
refers to areas in which gliosis was present and there was no sign of surviving neurons. A transparent grid of four $0.5 \times 0.5 \mathrm{~mm}$ squares was placed over each plate of the reconstructed lesions. A square was judged to have been destroyed if neuronal damage appeared in more than half of the $0.5 \mathrm{~mm}^{2}$ area. Bilaterally, the PPTg comprised 32 squares, and neuronal damage was described in terms of the number of squares that were destroyed.

\section{Statistical analysis}

Reinforced lever-pressing data were analyzed using a two-way repeated measures ANOVA with group as a nonrepeated factor and session as a repeated factor. A second repeated factor (time within session) was introduced into the analyses of extinction and reacquisition data. Post hoc analyses were conducted using Scheffé's test.

\section{RESULTS}

Pre-training lesions of the PPTg completely blocked the acquisition of heroin self-administration in more than half of the lesioned animals and reduced the effect in the remainder of the group. Lesioned animals that acquired self-administration on an FR-1 schedule showed normal patterns of responding during extinction and reacquisition but had reduced break points when tested on a PR schedule of reinforcement. Asymmetric lesions had no effect on responding under either schedule. Symmetric lesions that were made after self-administration training were also ineffective.

\section{Pre-training lesions \\ Acquisition}

Injections of the neurotoxin NMDA into the vicinity of the PPTg disrupted the acquisition of intravenous heroin selfadministration. Over the 15 sessions, animals with symmetric damage to the PPTg $(n=26)$ received fewer infusions of heroin than did sham-lesioned animals $(n=16)$ or animals with asymmetric lesions $(n=13)$ (interaction, $\left.F_{(28,728)}=4.728 ; p<0.0001\right)$. There was also a main effect of group $\left(F_{(2,728)}=13.317 ; p<\right.$ $0.0001)$ because of the fact that rats with symmetric PPTg lesions administered less heroin than sham-lesioned animals $\left(F_{(14,560)}=\right.$ 6.932; $p<0.0001)$. In contrast, the animals with asymmetric lesions (the majority of the PPTg destroyed in one, but not the other, nucleus) learned to self-administer heroin at the same rate as the sham-lesioned animals $\left(F_{(14,378)}=0.793 ; p=0.6766\right)$. Post hoc analysis indicated that the sham- and PPTg-lesioned animals received the same number of heroin infusions during the first three sessions, but that during sessions 4-15 lesioned animals made significantly fewer reinforced bar presses.

A closer examination of the behavioral data indicated that some animals with symmetric PPTg lesions increased their heroin intake over the 15 acquisition sessions. Consequently, the data from the 26 rats with histologically verified symmetric PPTg lesions were divided into two groups using the following criterion. The average number of heroin inf usions earned by the rats with sham lesions over the last $5 \mathrm{~d}$ of testing (sessions 11-15) was determined to be 15.038 with an SD of 4.798. Lesioned animals whose average drug intake over sessions 11-15 fell $>2$ SD below the mean of the sham-lesioned intake (i.e., $<5.442$ reinforced bar presses over the last $5 \mathrm{~d}$ of testing) were considered not to have learned to self-administer heroin. Lesioned rats whose average drug intake over the last $5 \mathrm{~d}$ of testing exceeded 5.442 infusions were considered to have learned the task.

Figure 1 shows that animals with "effective" PPTg lesions $(n=$ 14) were impaired in the acquisition of heroin self-administration (interaction, $\left.F_{(14,392)}=10.621 ; p<0.0001\right)$ and did not increase

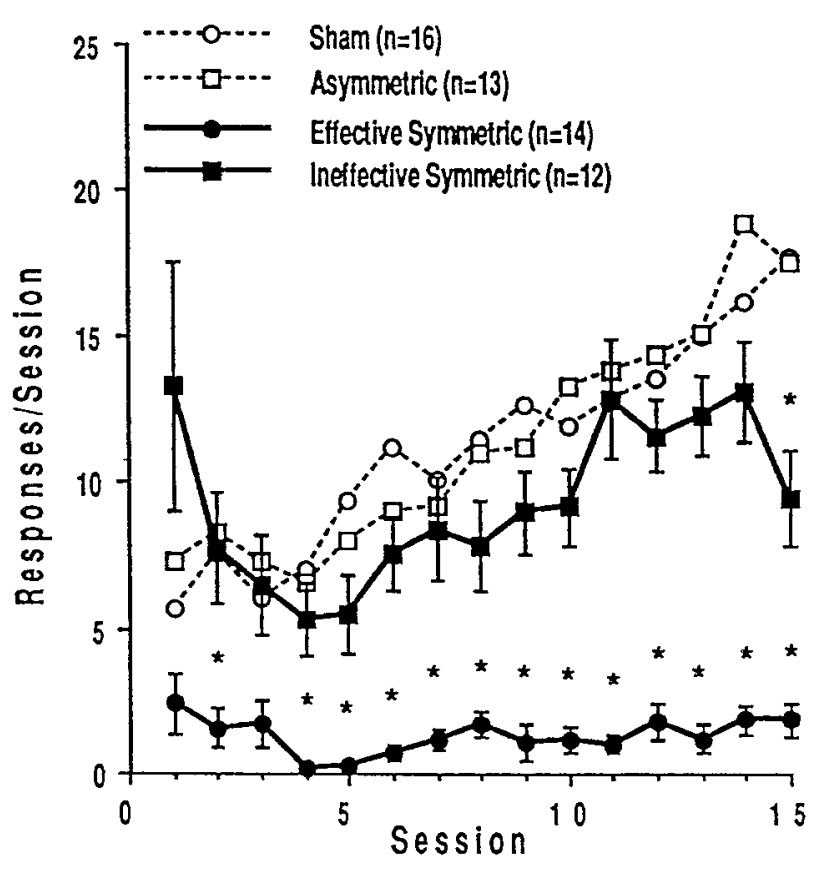

Figure 1. Mean \pm SEM numbers of heroin-reinforced lever presses for the first 15 training sessions in subgroups of the symmetric lesion condition. Effective lesions were defined as those that caused selfadministration rates 2 SD lower than those of the sham-lesioned animals (shown with those of the asymmetric-lesioned animals in dotted lines). Stars indicate data points significantly $(p<0.05)$ different from the corresponding point for the sham-lesioned animals.

their heroin intake over the 15 test sessions $\left(F_{(1,392)}=85.526 ; p<\right.$ 0.0001). Post hoc analyses indicated that these lesioned animals made significantly fewer reinforced bar presses than shamlesioned animals during each session with the exceptions of sessions 1 and 3. Figure 1 further illustrates that the animals with "ineffective" PPTg lesions $(n=12)$ increased their rate of heroin self-administration across testing but were still impaired compared with sham-lesioned animals (interaction, $F_{(14,364)}=3.791$; $p<0.0001)$. Although there was not a significant main effect of group (sham vs lesions) $\left(F_{(1,364)}=1.698 ; p=0.204\right)$, post hoc analysis indicated that PPTg-lesioned rats took significantly less drug than sham-lesioned rats during session 15. The average intake of PPTg-lesioned rats that acquired FR responding was greater than that of sham-lesioned rats during session 1 , but the difference was not significant because of the large variability in the lesioned group. Two rats took excessive amounts of heroin on the first day of testing (39 and 47 infusions, respectively) but did not continue to respond at such high rates over the next 14 sessions.

There was also a difference in the mean number of heroin infusions for each group summed over the 15 acquisition sessions (mean reinforced bar presses: sham lesions, $168.31 \pm 14.61$; PPTg lesions that did not acquire FR responding, $19.79 \pm 3.88$; PPTg lesions that did acquire FR responding, $139.58 \pm 16.39$; and asymmetric lesions, $170.92 \pm 19.03)$. Statistical analyses of the mean number of heroin infusions across acquisition sessions revealed a significant effect of group $\left(F_{(3,54)}=25.045 ; p<0.0001\right)$ and significant post hoc differences between the total intake of lesioned animals that did not learn the response and every other group. 


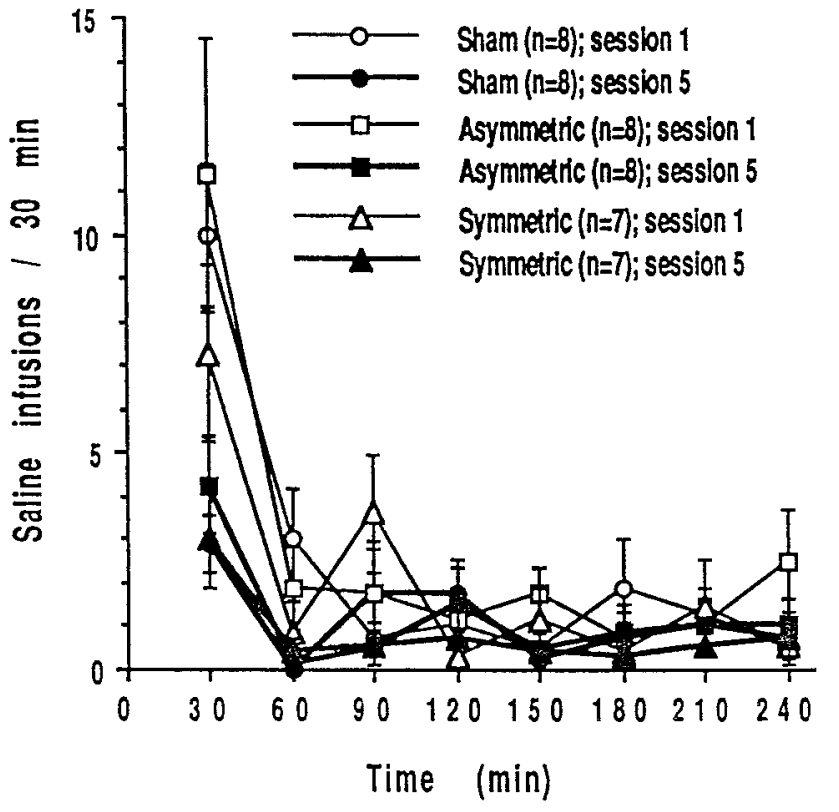

Figure 2. Mean \pm SEM numbers of responses during extinction when saline was given in place of heroin on days $16-20$.

\section{Extinction}

After FR1 training, seven lesioned rats that acquired FR responding, eight animals with asymmetric lesions, and eight animals with sham lesions were tested for an additional five sessions during extinction. There were no significant differences in the response patterns of these three groups over five $4 \mathrm{hr}$ extinction sessions (interaction, group $\times$ session $\times$ time, $F_{(14,140)}=0.685 ; p=$ $0.7859)$. Typically, when responding is not reinforced, an animal will initially increase its responding, but the behavior will extinguish quite rapidly during the first session. On subsequent sessions, extinction occurs more rapidly so that the increase in responding at the beginning of a session is not as marked. Figure 2 shows that animals with sham, asymmetric, and PPTg lesions displayed this pattern of responding. Statistical analyses reflect the observation; there was a significant session $\times$ time interaction $\left(F_{(7,308)}=9.282 ; p=0.0001\right)$ but no main effect of group $\left(F_{(2,20)}\right.$ $=1.249 ; p=0.3082)$, and in all three groups response rates were higher in the first than in the fifth extinction session $\left(F_{(1,308)}=\right.$ $14.461 ; p=0.0004)$. Post hoc analysis revealed that the difference in response rates between days 1 and 5 occurred at 30 and $60 \mathrm{~min}$ intervals but not during the remaining $3 \mathrm{hr}$ of the sessions.

\section{Reacquisition}

After extinction tests, animals were allowed to self-administer heroin for an additional five sessions. Because of blocked catheters and lost head caps, seven animals were eliminated from behavioral testing, leaving four animals with symmetric lesions, five animals with asymmetric lesions, and seven animals with sham lesions (data not shown). All of these animals quickly reacquired heroin self-administration. In fact, the pattern of responding during a $4 \mathrm{hr}$ self-administration session on the 5th day of access to heroin (after extinction) was the same as it was on the 15th day of the initial acquisition sessions for animals with symmetric, asymmetric, and sham lesions of the PPTg (group $\times$ session $\times$ time, $\left.F_{(14,91)}=1.120 ; p=0.3446\right)$, and there was no main effect of group $\left(F_{(2,13)}=0.22 ; p=0.9784\right)$. In both acquisition and reacquisition sessions, there was an initial loading

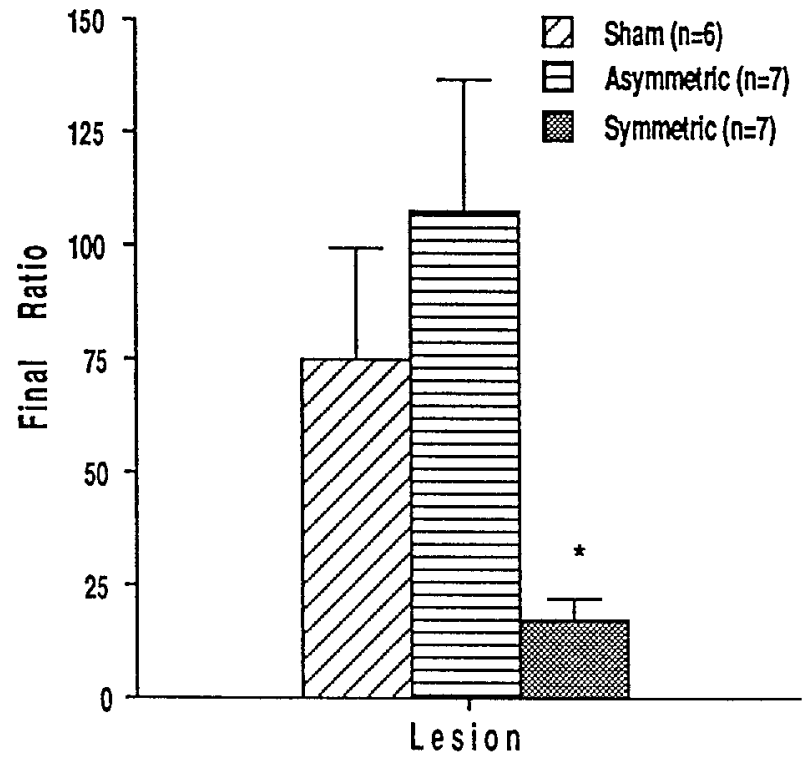

Figure 3. Final response ratios \pm SEM for intravenous heroin on the progressive ratio schedule of reinforcement in rats lesioned before initial training.

phase at the beginning of the session, and then intake was maintained at a steady rate for the remainder of the session. Although the difference was not statistically significant, heroin intake of PPTg-lesioned rats was less than that of sham-lesioned rats on the fifth day of reacquisition.

\section{$P R$ schedule of reinforcement}

Twenty rats (seven with symmetric, seven with asymmetric, and six with sham lesions) that had learned to self-administer intravenous heroin on an FR-1 schedule during the initial 15 acquisition sessions were tested on a PR schedule of reinforcement. Break points of all animals stabilized within $8.05 \pm 0.71 \mathrm{~d}$ (sham lesions, $7.0 \pm 1.63 \mathrm{~d}$; symmetric lesions, $7.14 \pm 0.34 \mathrm{~d}$; asymmetric lesions, $9.29 \pm 1.63 \mathrm{~d}$ ). The final ratios (number of bar presses made for the last infusion of heroin) and the ordinal values of these final ratios (progressive ratio step number) are shown in Figure 3. Rats with PPTg lesions stopped responding at a lower step in the progressive ratio schedule than sham-lesioned rats; that is, lesioned animals made fewer bar presses for their final infusion of heroin $\left(F_{(2,17)}=11.885 ; p=0.0006\right)$. Break points of rats with sham and asymmetric lesions of the PPTg were not significantly different.

\section{Passive heroin injections}

Four rats with symmetric, effective PPTg lesions that failed to learn to lever press (FR-1) for intravenous heroin within $15 \mathrm{~d}$ were subsequently treated with daily heroin injections $(0.5 \mathrm{mg} / \mathrm{kg}$, s.c.) for $4 \mathrm{~d}$ and then retrained. After passive drug exposure, these animals learned to lever press (FR-1) for heroin at the same rate as did drug-naive animals with sham lesions (Fig. 4). There was no significant difference in response rates across 15 sessions of drugnaive animals and of PPTg-lesioned animals that were previously treated with heroin injections (main effect of group, $F_{(1,17)}=0.64$; $p=0.83$; group $\times$ session interaction, $\left.F_{(14,238)}=0.64 ; p=0.83\right)$. The response rates of PPTg-lesioned animals over 15 sessions were significantly altered by the heroin injections; there was a significant main effect of group $\left(F_{(1,6)}=22.74 ; p=0.008\right)$ and of 


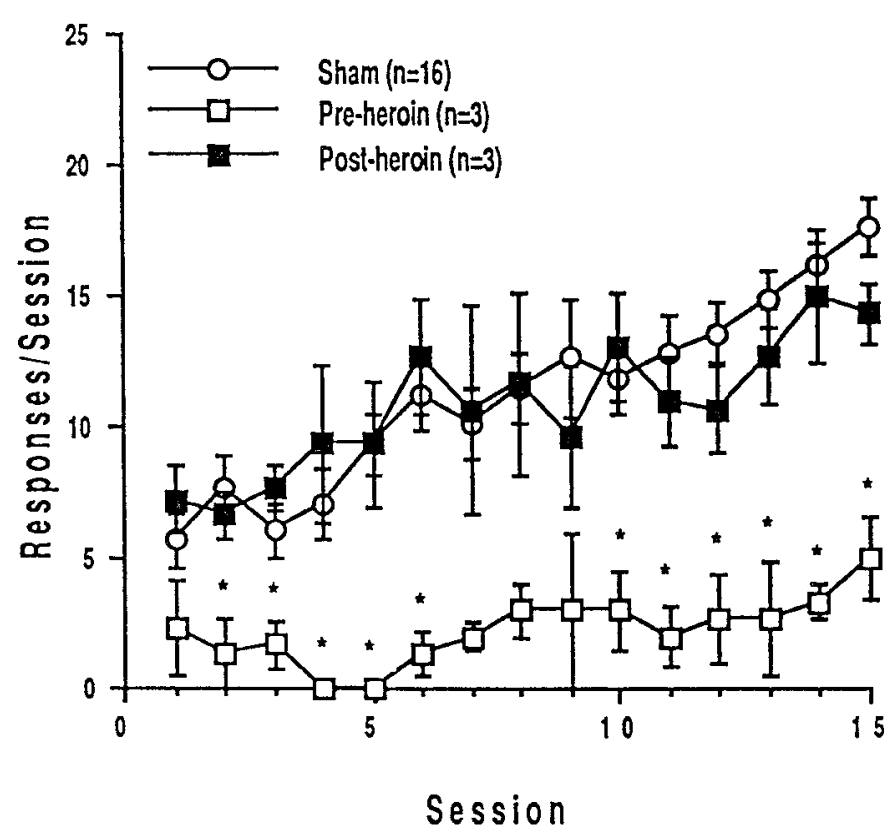

Figure 4. Mean \pm SEM numbers of heroin-reinforced lever presses over 15 training sessions before and after four daily injections of heroin in rats with sham or effective PPTg lesions.

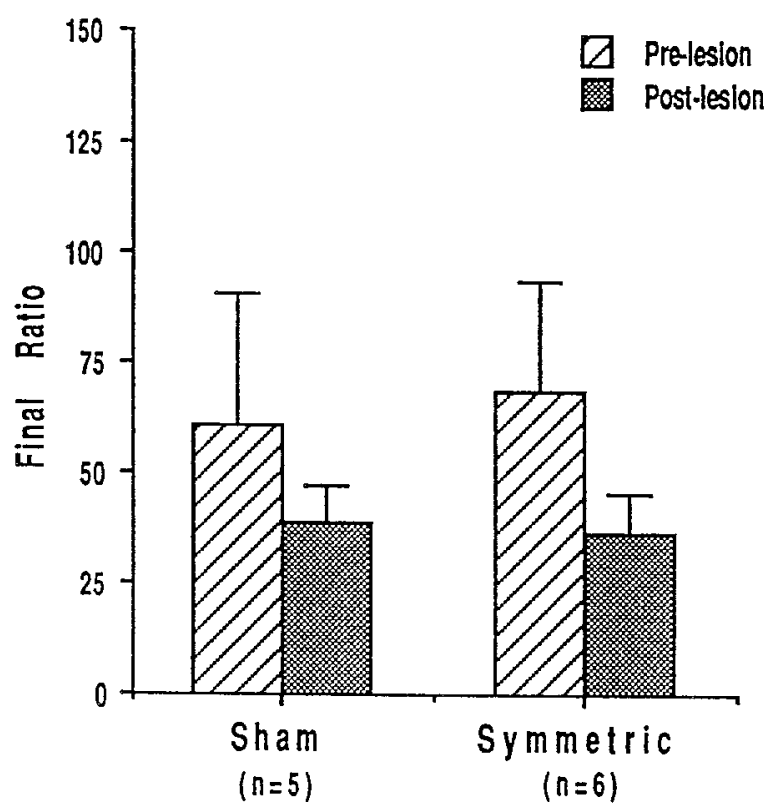

Figure 5. Final response ratios \pm SEM for intravenous heroin on the progressive ratio schedule of reinforcement in rats lesioned after response acquisition and progressive ratio training.

session $\left(F_{(14,56)}=2.852 ; p=0.0027\right)$ but no significant group $\times$ session interaction $\left(F_{(14,56)}=1.117 ; p=0.364\right)$.

\section{Post-training lesions}

Animals that were implanted with bilateral cannulae and lesioned after self-administration training showed no deficit in responding for heroin on a PR schedule of reinforcement (Fig. 5). Sham $(n=$ $5)$ and lesioned $(n=6)$ animals had the same break points, that is they made approximately the same number of bar presses for their final infusion of heroin $\left(F_{(1,9)}=0.01 ; p=0.9218\right)$. Furthermore, there was no significant difference between prelesion and postlesion break points in either group of animals (interaction, $F_{(1,9)}=0.601, p=0.458$; main effect of session, $F_{(1,9)}=3.562 ; p=$ 0.0917 ; and main effect of group, $\left.F_{(1,9)}=0.01 ; p=0.9218\right)$.

\section{Histology}

PPTg lesions were concentrated in the subnucleus compactus region of the nucleus, dorsolateral to the superior cerebellar peduncle (Figs. 6, 7). Cell loss or gliosis was present in the ventromedial PPTg in one-third of the lesioned animals. Neurotoxin-induced damage extended throughout the anteriorposterior plane of the PPTg, although there were no cases in which the entire PPTg was destroyed in a single subject. In some animals, there was partial destruction of the cuneiform nucleus, the mesencephalic reticular nucleus, the retrorubral area, or the parabrachial area. Surrounding regions, including the PAG, the VTA, and the SN, were all spared. The animals with symmetric lesions had the majority of the PPTg destroyed in both hemispheres; those with asymmetric lesions had the majority of the PPTg destroyed in one, but not the other, nucleus.

The behavioral deficits exhibited by animals with pre-training, symmetric PPTg lesions varied. Based on their effectiveness in blocking acquisition of FR-1 responding (using the criterion specified above), the lesions were classified as effective or ineffective. Neuronal destruction and cholinergic cell loss within subregions of the PPTg were measured in effective, ineffective, and sham-lesioned brains.

Neuronal destruction within the PPTg (cholinergic and noncholinergic) was measured by counting the numbers of $0.5 \mathrm{~mm}$ squares that were destroyed on plates of the reconstructed lesions. The majority of the PPTg was destroyed bilaterally in both groups, but there were differences between the location of neuronal damage within the nucleus. Specifically, the mean number of damaged squares was similar for animals with effective (26 \pm $3.12)$ and ineffective $(23 \pm 4.43)$ lesions. In contrast, all effective lesions destroyed at least 14 of the 16 posterior squares, whereas only 8-13 squares were destroyed in the posterior half of the PPTg after ineffective lesions. Thus, lesions that blocked FR-1 acquisition were consistently concentrated in the caudal regions of the nucleus, producing cell damage across the dorsoventral plane at the posterior end of the nucleus. Half of the animals in this group also had neuronal damage in the ventromedial PPTg. There was more variation in the location of lesions that did not completely block FR acquisition, but in general they destroyed neurons in the rostral PPTg but spared those in the caudal-most regions. These lesions also produced damage in both the dorsolateral and ventromedial PPTg.

Cholinergic cell loss was quantified by counting the number of NADPH-diaphorase-stained cells in each hemisphere of animals with effective and ineffective lesions and comparing these with the cell loss in a subpopulation $(n=10)$ of sham-lesioned brains. Sham-lesioned brains contained a mean of $1489 \pm 103$ neurons per hemisphere, a figure that is consistent with previous descriptions of PPTg morphology (Rugg et al., 1992; Olmstead and Franklin, 1993). The numbers of ACh neurons were greatly reduced in both animals with effective $(193.57 \pm 69)$ and ineffective $(357.36 \pm 81)$ lesions. This corresponds to 87 and $76 \%$ loss of cholinergic cells in the two groups of animals. Lesions produced a statistically significant loss of cholinergic neurons $\left(F_{(2,33)}=\right.$ 169.54; $p<0.001)$, but post hoc comparisons indicated that there was no significant difference between the number of cholinergic neurons remaining in the effective and ineffective lesioned groups. Cholinergic cell loss within the PPTg was compared by 

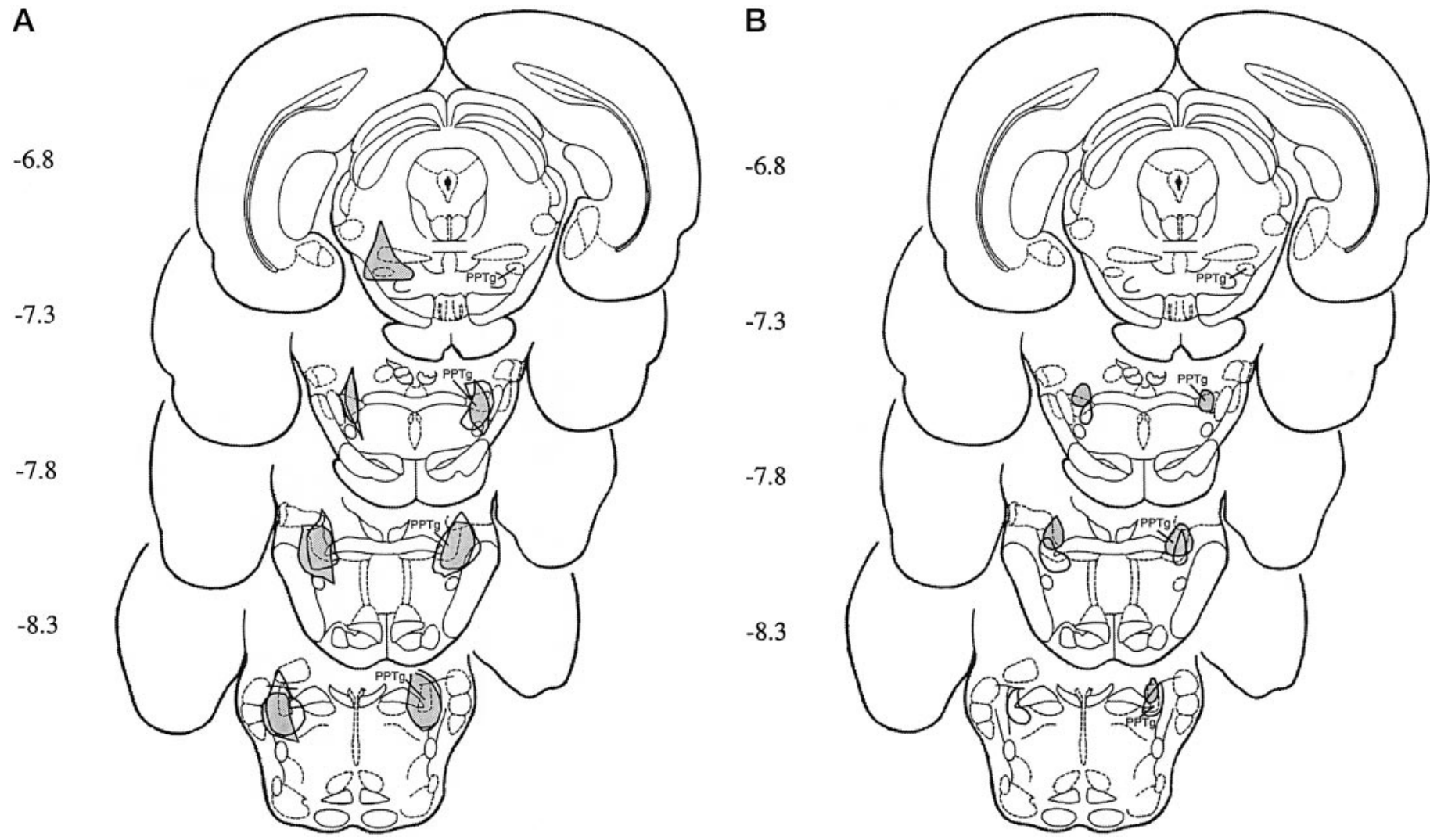

Figure 6. Histological localization of PPTg lesions in animals with the largest $(A)$ and smallest $(B)$ effective (open area) and ineffective (shaded area) lesions.

counting the number of NADPH-diaphorase-stained cells that remained in the 16 anterior and 16 posterior $0.5 \mathrm{~mm}$ squares. Lesions produced a significant loss of cholinergic neurons in the anterior $\left(F_{(2,33)}=14.56 ; p<0.05\right)$ and posterior $\left(F_{(2,33)}=129.68\right.$; $p<0.01)$ PPTg. Post hoc tests showed that effective lesions destroyed more cholinergic neurons in the posterior PPTg than did ineffective lesions, whereas the loss of cholinergic neurons in the anterior PPTg was not significantly different in the two lesioned groups.

In both effective and ineffective lesioned groups, the neuronal damage extended beyond the boundaries of the PPTg, but none of the non-PPTg structures were consistently destroyed in either group. For example, in the effective group seven of the animals had partial damage in the cuneiform nucleus, two in the lateral dorsal tegmental nucleus, five in the parabrachial area, and 10 in the medial region of the PPTg ventral to the decussation of the superior peduncle. In the ineffective group, nine animals had partial damage in the retrorubral area, six in the cuneiform nucleus, five in the mesencephalic reticular nucleus, four in the parabrachial area, seven in the subpeduncular tegmental nucleus, and one in the lateral dorsal tegmental nucleus.

\section{DISCUSSION}

PPTg lesions that were both bilateral and symmetrical reduced the rewarding effect of self-administered heroin. One group of animals with symmetric PPTg lesions did not acquire heroin self-administration over $15 \mathrm{~d}$ of training on an FR-1 schedule. A second group of PPTg-lesioned animals exhibited minimal deficits in the acquisition sessions and learned the response requirements of the PR schedule but had lower break points than sham-lesioned animals. Our finding that PPTg lesions produce motivational deficits is consistent with previous evidence that the PPTg contributes in some way to the rewarding effect of drugs and food in the CPP paradigm (Bechara and van der Kooy, 1989, 1992; Olmstead and Franklin, 1993, 1994a).

The effectiveness of symmetric PPTg lesions on the acquisition of heroin self-administration varied dramatically. Indeed, animals with symmetric PPTg lesions appeared to exhibit one of two distinct patterns of responding across the 15 training sessions. Fourteen of the 26 lesioned animals increased their heroin intake across sessions (although to a lesser extent than did the shamlesioned animals), whereas heroin intake of the remaining lesioned animals was no higher on the 15 th session than on the 1 st test session (Fig. 1). All animals with symmetric lesions did emit reinforced lever presses on several occasions during testing, suggesting that the failure of some animals to acquire heroin selfadministration did not reflect a performance deficit that prevented them from making the required response. Furthermore, a subset of these lesioned animals responded at the same level as sham-lesioned animals on an FR-1 schedule after passive heroin infusions.

The behavioral differences in response acquisition between animals with symmetric lesions are probably not attributable to differences in the amount of neurotoxin-induced damage but may be related to its localization. There was no significant difference in either the total volume of neuronal damage or in the reduction in numbers of PPTg cholinergic neurons in lesions associated with acquisition or failed acquisition of the response habit. These findings are consistent with our previous report that the blockade 

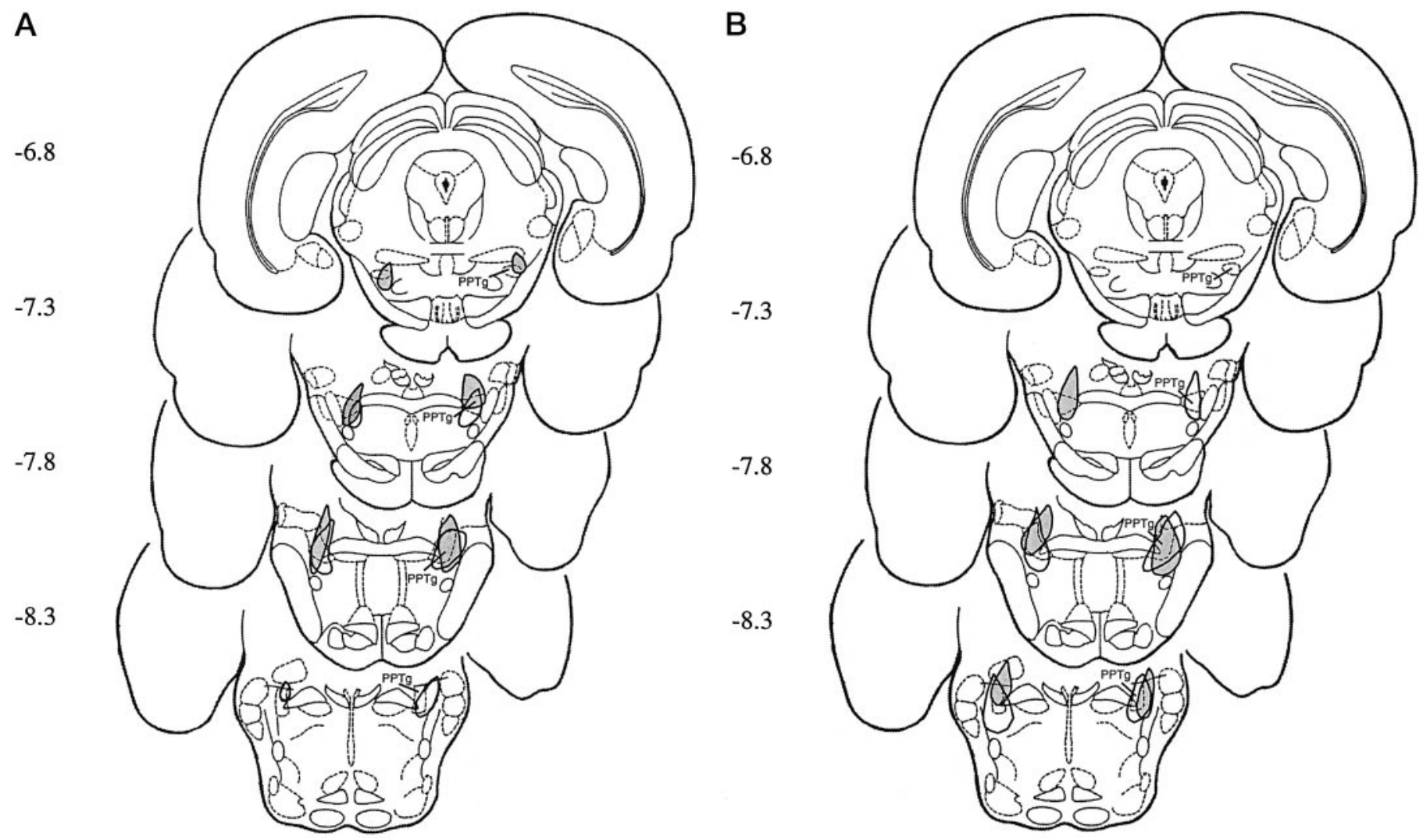

Figure 7. Histological localization of PPTg lesions in animals with the most anterior $(A)$ and the most posterior $(B)$ effective (open area) and ineffective (shaded area) lesions.

of a morphine-induced CPP is not related to the magnitude of cholinergic cell loss in the PPTg (Olmstead and Franklin, 1993). The animals exhibiting the most pronounced acquisition deficits in the present study, however, had a greater degree of cholinergic and noncholinergic neuronal destruction in the caudal PPTg. This neurological difference between the two groups may be the cause of the behavioral difference in the FR-1 acquisition sessions. Our results do not clarify whether the PPTg involvement in reward is mediated through its ascending cholinergic (Yeomans, 1995) or its descending noncholinergic (Bechara and van der Kooy, 1989) projections. Pontomesencephalic cholinergic projections to the VTA originate in the caudal PPTg as well as in the lateral-dorsal tegmental nucleus (Oakman et al., 1995b). Although effective lesions produced a greater loss of cholinergic neurons in the caudal PPTg, they also consistently damaged more noncholinergic neurons in this region than did ineffective lesions. It is clear that the PPTg is a heterogeneous nucleus containing different subpopulations of neurons that are distributed unevenly across the nucleus; our results indicate that this anatomical distinction may be reflected in a functional heterogeneity within the nucleus.

PPTg lesions produce subtle abnormalities in sensorimotor tests (Dunbar et al., 1992; Olmstead and Franklin, 1994b), but it is unlikely that lesion-induced deficits in the present study reflected a motor impairment. First, post-training lesions did not affect response rates on either fixed or progressive ratio schedules of reinforcement. Second, lesioned animals were clearly capable of responding at rates higher than those exhibited during acquisition; animals lesioned before training that subsequently acquired responding on an FR-1 schedule exhibited accelerated rates of responding during extinction. Moreover, a subset of lesioned animals that did not acquire self-administration responded at the same level as sham-lesioned animals on an FR1 schedule after four passive infusions of heroin. Finally, animals with PPTg lesions induced using the same parameters respond at much higher rates for food (Olmstead et al., 1995) or electrical brain stimulation (Munn et al., 1994).

Nor can a simple learning deficit account for the difference between pre- and post-training lesions. Although the rate of acquisition and level of responding were reduced after PPTg lesions, some lesioned animals learned the lever pressing response on an FR-1 schedule and were capable of performing on a PR schedule. Similarly, PPTg lesions do not block the acquisition of an operant response for sucrose reward on either FR or PR schedules (Olmstead et al., 1995). The fact that sham- and PPTglesioned animals exhibited similar rates and patterns of responding during extinction and reacquisition also suggests that PPTg lesions do not disrupt the learning processes that mediate operant responding.

Asymmetric lesions (defined as destruction of the majority of the PPTg in only one hemisphere) may be thought of as unilateral lesions. The ineffectiveness of these lesions therefore appears, on the surface at least, to be inconsistent with the reported finding that unilateral destruction of the PPTg decreases the rewarding effect of electrical stimulation of the contralateral LH (Buscher et al., 1989). The critical difference between the two studies is probably that neuronal destruction in the study by Buscher et al. (1989) did not encroach on the caudal PPTg, whereas our most effective lesions were concentrated in this region. PPTg lesions in the study by Buscher et al. (1989) also destroyed the retrorubral 
field, and, although lesions of the retrorubral field alone are ineffective, contralateral damage to this area may contribute to the deficits induced by PPTg lesions in the ICS paradigm (Lepore and Franklin, 1996).

Post-training lesions of the PPTg also had no effect on responding for intravenous heroin on either FR or PR schedules of reinforcement. The disruption in acquisition, but not maintenance, of heroin self-administration is consistent with the findings that a CPP to opiates is blocked by preconditioning, but not postconditioning, lesions of the PPTg (Bechara and van der Kooy, 1989) and that rates of heroin self-administration do not change after PPTg lesions (Nader et al., 1994). The lack of effectiveness of post-training lesions is not contrary to our suggestion that PPTg lesions produce a motivational deficit, because posttraining reductions in the incentive value of a stimulus contingent upon an operant response are relatively ineffective in well trained animals (Adams, 1982). For example, Yokel and Pickens (1976) have demonstrated that the pattern of responding for intravenous stimulants that is acquired during training continues in extinction. That is, with the initial removal of the rewarding stimulus, conditioned patterns of responding persist for a substantial period. To explain this phenomenon, it has been suggested that during the early stages of operant training, behavior is controlled by a response-reinforcer expectancy, but with repeated training a stimulus-response habit develops such that devaluation of the reinforcer has less effect on responding (Bolles, 1972; Adams and Dickinson, 1981).

On the other hand, the ineffectiveness of post-training lesions may be related, not to experience with the instrumental contingency, but to the level of drug exposure. Bechara and colleagues (Bechara et al., 1992; Bechara and van der Kooy, 1992) have proposed that PPTg lesions block the rewarding effect of drugs when animals are drug-naive but not when they are drugdependent. Our finding that lesioned animals that did not previously acquire the self-administration habit do so after a series of experimenter-administered heroin injections is the strongest evidence to date for this hypothesis. No doubt, one or more of the neural substrates that mediate reward, perhaps even elements within the PPTg, have been altered by repeated drug administration either in the prelesion training sessions or by the experimenter-administered heroin injections such that the reward deficits are no longer apparent.

Bechara and colleague's proposed dissociation between the neural systems that mediate reward in drug-naive and drugexperienced animals (Bechara et al., 1992; Bechara and van der Kooy, 1992) could also be used to explain the behavioral difference between the effectiveness of pre-training PPTg lesions. If lesioned animals that acquired responding on the FR schedule had high levels of responding during the initial acquisition sessions, their heroin intake (and subsequent drug experience) would have been greater than that of animals that did not acquire FR responding. According to Bechara et al. (1992) and Bechara and van der Kooy (1992), animals with the history of increased heroin intake in the initial sessions would continue to selfadminister to alleviate the aversive effects of opiate abstinence. Although we cannot rule out this suggestion, we did not observe behavioral effects that support it. Heroin intake of lesioned animals that acquired self-administration under the FR-1 schedule was anomalously high during session 1 (Fig. 1) because of two animals that did not continue to self-administer at high rates during the remaining acquisition sessions. Furthermore, there was no relationship between heroin intake on days 1 and 15 in lesioned animals, indicating that the amount of drug exposure in the initial session did not predict whether the animals would or would not continue to self-administer. Finally, the idea that drug experience distinguishes animals that did from the animals that did not acquire the habit does not explain why lesioned animals that acquired responding exhibited a motivational deficit when tested on a PR schedule of reinforcement. Moreover, the hypothesis that PPTg lesions only reduce the rewarding effect of the drug when animals are drug-naive does not fit well with the evidence that PPTg lesions disrupt performance in a variety of learning and memory tasks. That is, the effectiveness of PPTg lesions does not simply depend on animals being in a nondeprived or motivationally neutral state; learning deficits induced by PPTg lesions are still apparent when animals are in a state of deprivation (Kessler et al., 1986; Dellu et al., 1991; Lepore, 1993; Inglis et al., 1994).

Our suggestion that PPTg lesions disrupt rewarding processes does not discount a role for the nucleus in cognitive functions. PPTg-lesioned animals are clearly capable of forming simple associations (Bechara et al., 1992; Bechara and van der Kooy, 1992; Guarraci et al., 1994), but learning deficits become apparent in more complex tasks (Kessler et al., 1986; Dellu et al., 1991). Lesioned animals in the present study did not exhibit a learning deficit, but there was only one lever in the operant chamber; if a second lever were introduced, it is likely that the animals would be unable to distinguish between the levers associated with reward and nonreward (Inglis et al., 1994). The PPTg involvement in learning may be specifically related to the role of this structure in attention, mediated through its participation in the reticular activating and thalamocortical systems (Fitzpatrick et al., 1989; Steriade et al., 1990) and by regulation of forebrain ACh systems (Bertorelli et al., 1991). If the PPTg is involved in both reward and attention, information about the two processes may be integrated within the nucleus. Deficits after PPTg lesions, therefore, could be attributable not to an attenuation of rewarding or attentional processes but to some interaction of the two. The fact that the cholinergic projections from the PPTg collateralize to the VTA and thalamus (Oakman et al., 1995a) is consistent with this hypothesis and could suggest that PPTglesioned animals are unable to attend to stimuli associated with reward when the demands of the task are increased.

\section{REFERENCES}

Adams CD (1982) Variations in the sensitivity of instrumental responding to reinforcer devaluation. Q J Exp Psychol 34B:77-98.

Adams CD, Dickinson A (1981) Actions and habits: variations in associative representations during instrumental learning. In: Information processing in animals: memory mechanisms (Spear NE, Miller RR, eds). Hillsdale, NJ: Erlbaum.

Bechara A, van der Kooy D (1989) The tegmental pedunculopontine nucleus: a brain-stem output of the limbic system critical for the conditioned place preferences produced by morphine and amphetamine. J Neurosci 9:3400-3409.

Bechara A, van der Kooy D (1992) A single brainstem substrate mediates the motivational effects of both opiates and food in non-deprived rats but not in deprived rats. Behav Neurosci 106:351-363.

Bechara A, Harrington F, Nader K, van der Kooy D (1992) Neurobiology of motivation: double dissociation of two motivational mechanisms mediating opiate reward in drug-naive versus drug-dependent animals. Behav Neurosci 106:798-807. 
Bertorelli R, Forloni G, Consolo S (1991) Modulation of the cortical in vivo ACh release by the basal nuclear complex: role of the pontomesencephalic tegmental area. Brain Res 563:353-356.

Bolles RC (1972) Reinforcement, expectancy, and learning. Psychol Rev 79:394-409.

Buscher W, Schugens M, Wagner U, Huston JP (1989) Interhemispheric relationship between lateral hypothalamic self-stimulation and the region of the nucleus tegmenti pedunculo-pontinus. Brain Res 487:321-334.

Dellu F, Mayo W, Cherkauoui J, LeMoal M, Simon H (1991) Learning disturbances following excitotoxin lesion of cholinergic pedunculopontine nucleus in the rat. Brain Res 544:126-132.

Dunbar JS, Hitchcock K, Latimer M, Rugg EL, Ward N, Winn P (1992) Excitotoxin lesions of the pedunculopontine tegmental nucleus of the rat II. Examination of eating and drinking, rotation, and reaching and grasping following unilateral ibotenate or quinolinate lesions. Brain Res 589:194-206.

Fitzpatrick D, Diamond IT, Raczkowski D (1989) Cholinergic and monoaminergic innervation of the cat's thalamus: comparison of the lateral geniculate nucleus with other principal sensory nuclei. J Comp Neurol 288:647-675.

Fujimoto K, Yoshida M, Ikeguchi K, Niijima K (1989) Impairment of active avoidance produced after destruction of pedunculopontine nucleus areas in the rat. Neurosci Res 6:321-328.

Fujimoto K, Ikeguchi K, Yoshida M (1992) Impaired acquisition, preserved retention and retrieval of avoidance behavior after destruction of pedunculopontine nucleus areas in the rat. Neurosci Res 13:43-51.

Garcia-Rill E, Skinner RD, Gilmore SA, Owings R (1983) Connections of the mesencephalic locomotor region (MLR) II. Afferents and efferents. Brain Res Bull 10:63-71.

Glickman SE, Schiff BB (1967) A biological theory of reinforcement. Psychol Rev 74:81-109.

Guarraci F, Melanson N, Franklin KBJ, Olmstead MC (1994) Effects of PP Tg lesions on the conditioned locomotor response to stimulants. Soc Neurosci Abstr 20:1029.

Hallanger AE, Levey AI, Lee HJ, Rye DB, Wainer BH (1987) The origins of cholinergic and other subcortical afferents to the thalamus in the rat. J Comp Neurol 262:105-124.

Hallanger AE, Wainer BH (1988) Ascending projections from the pedunculopontine tegmental nucleus and the adjacent mesopontine tegmentum in the rat. J Comp Neurol 274:483-515.

Herberg JL, Franklin KBJ (1972) Adrenergic feeding: its blockade or reversal by posterior VMH lesions; and a new hypothesis. Physiol Behav 8:1029-1034.

Inglis WL, Winn P (1995) The pedunculopontine tegmental nucleus: where the striatum meets the reticular formation. Prog Neurobiol 47:1-29.

Inglis WL, Dunbar JS, Winn P (1994) Outflow from the nucleus accumbens to the pedunculopontine tegmental nucleus: a dissociation between locomotor activity and the acquisition of responding for conditioned reinforcement stimulated by D-amphetamine. Neuroscience 62:51-64.

Keesey RE, Powley TL (1973) Self-stimulation and body weight in rats with lateral hypothalamic lesions. Am J Physiol 224:970-978.

Kessler J, Markowitsch HJ, Sigg G (1986) Memory related role of the posterior cholinergic system. J Neurosci 30:101-119.

Klitenick MA, Kalivas PW (1994) Behavioral and neurochemical studies of opioid effects in the pedunculopontine nucleus and mediodorsal thalamus. J Pharmacol Exp Ther 269:437-448.

Kofman O, Yeomans JS (1989) Cholinergic antagonists in ventral tegmentum elevate thresholds for lateral hypothalamic and brainstem self-stimulation. Pharmacol Biochem Behav 31:547-559.

Kofman O, McGlynn SM, Olmstead MC, Yeomans JS (1990) Differential effects of atropine, procaine and dopamine in the rat ventral tegmentum on lateral hypothalamic rewarding brain stimulation. Behav Brain Res 38:55-68.

Lepore M (1993) Parametric and neurological studies of brain stimulation reward. PhD thesis, McGill University.

Lepore M, Franklin KBJ (1996) N-Methyl-D-aspartate lesions of the pedunculopontine nucleus block acquisition and impair maintenance of responding reinforced with brain stimulation. Neuroscience $71: 147-155$.

Mogenson GJ (1987) Limbic-motor integration. Prog Psychobiol Physiol Psychol 12:117-170.
Mogenson GJ (1990) Brainstem systems for the control of behavioral acts. In: Brainstem mechanisms of behavior (Klemm WR, Vertes RP, eds), pp 171-195. New York: Wiley.

Mogenson GJ, Jones DL, Yim CY (1980) From motivation to action: functional interface between the limbic system and the motor system. Prog Neurobiol 14:69-97.

Mogenson GJ, Yang CR (1991) The contribution of basal forebrain to limbic-motor integration and the mediation of motivation to action. Adv Exp Med Biol 295:267-290.

Munn EM, Olmstead MC, Franklin KBJ, Wise RA (1994) The effects of lesions of the pedunculopontine nucleus (PPN) on brain stimulation reward (BSR). Soc Neurosci Abstr 20:558.

Nader K, Bechara A, Roberts DCS, van der Kooy D (1994) Neuroleptics block high- but not low-dose heroin place preferences: Further evidence for a two-system model of motivation. Behav Neurosci 108:1128-1138.

Newman DB, Ginsberg CY (1994) Brain-stem reticular nuclei that project to the thalamus in rats: a retrograde tracer study. Brain Behav Evol 44:1-39.

Oakman SA, Faris PL, Cozzari C, Hartman BK (1995a) Evidence that pontomesencephalic cholinergic projections to midbrain dopaminergic groups are a subset of the tegmentothalamic projection. Soc Neurosci Abstr 21:68.

Oakman SA, Faris PL, Kerr PE, Cozzari C, Hartman BK (1995b) Distribution of pontomesencephalic cholinergic neurons projecting to substantia nigra differs significantly from those projecting to ventral tegmental area. J Neurosci 15:5859-5869.

Olmstead MC, Franklin KBJ (1993) Effects of pedunculopontine tegmental nucleus lesions on morphine-induced conditioned place preference and analgesia in the formalin test. Neuroscience 57:411-418.

Olmstead MC, Franklin KBJ (1994a) Lesions of the pedunculopontine tegmental nucleus block drug-induced reinforcement but not amphetamine-induced locomotion. Brain Res 638:29-35.

Olmstead MC, Franklin KBJ (1994b) Lesions of the pedunculopontine tegmental nucleus abolish catalepsy and locomotor depression induced by morphine. Brain Res 662:134-140.

Olmstead MC, Eramian DR, Purdy DA, Franklin KBJ (1995) Effects of PPTg lesions on responding for sucrose reward on a progressive ratio schedule of reinforcement. Soc Neurosci Abstr 21:449.

Orlovsky GN (1970) Activity of reticulospinal neurons during locomotion. Biophys J 15:728-737.

Paxinos G, Watson C (1986) The rat brain in stereotaxic coordinates. New York: Academic.

Roberts DCS, Bennett SAL (1993) Heroin self-administration in rats under a progessive ratio schedule of reinforcement. Psychopharmacology 111:215-218.

Rugg EL, Dunbar JS, Latminer M, Winn P (1992) Comparison of the neurotoxic properties of various excitotoxins in the pedunculopontine tegmental nucleus of the rat, with particular reference to the loss of immunohistochemically identified cholinergic neurons. Brain Res 589:1-13.

Rye DB, Saper CB, Lee HJ, Wainer BH (1987) Pedunculopontine tegmental nucleus of the rat: cytoarchitecture, cytochemistry, and some extrapyramidal connections of the mesopontine tegmentum. J Comp Neurol 259:483-528.

Steeves JD, Jordan LM (1984) Autoradiographic demonstration of the projections from the mesencephalic locomotor region. Brain Res 307:263-276.

Steriade M, Datta S, Pare D, Oakson S, Curro R (1990) Neuronal activities in brain-stem cholinergic nuclei related to tonic activation processes in thalamocortical systems. J Neurosci 10:2541-2559.

Sugimoto T, Hattori T (1984) Organization and efferent projections of nucleus tegmenti pedunculopontinus pars compacta with special reference to its cholinergic aspects. Neuroscience 11:931-946.

Swanson LW, Cowan WM (1975) A note on the connections and development of the nucleus accumbens. Brain Res 92:324-330.

Swanson LW, Mogenson GJ, Gerfen CR, Robinson P (1984) Evidence for a projection from the lateral preoptic area and substantia innominata to the mesencephalic locomotor region in the rat. Brain Res 295:161-178.

Trojniar W, Staszewska M (1995) Bilateral lesions of the pedunculopontine tegmental nucleus affect feeding induced by electricalstimulation of the ventral tegmental area. Acta Neurobiol Exp 55:201-206. 
Tsai CT, Mogenson GJ, Wu M, Yang CR (1989) A comparison o the effects of electrical stimulation of the amygdala and hippocampus on subpallidal output neurons to the pedunculopontine nucleus. Brain Res 494:22-29.

Vincent SR, Satoh K, Armstrong DM, Fibiger HC (1983) NADPHdiaphorase: a selective histochemical marker for the cholinergic neurons of the pontine reticular formation. Neurosci Lett 43:31-36.

Waraczynski M, Shizgal P (1995) self-stimulation of the MFB following parabrachial lesion. Physiol Behav 58:559-566.

Woolf NJ, Butcher LL (1986) Cholinergic systems in the rat brain: III. Projections from the pontomesencephalic tegmentum to the thalamus, tectum, basal ganglia, and basal forebrain. Brain Res Bull 16:603-637.
Yeomans JS (1995) Role of tegmental cholinergic neurons in dopaminergic activation, antimuscarinic psychosis and schizophrenia. Neuropsychopharmacology 12:3-16.

Yeomans JS, Kofman O, McFarlane V (1985) Cholinergic involvement in lateral hypothalamic rewarding brain stimulation. Brain Res 329:19-26.

Yeomans JS, Mathur A, Tampakeras M (1993) Rewarding brain stimulation: role of tegmental cholinergic neurons that activate dopamine neurons. Behav Neurosci 107:1077-1087.

Yokel RA, Pickens R (1976) Extinction responding following amphetamine self-administration: determination of reinforcement magnitude. Physiol Psychol 4:39-42. 